\title{
Incentivo paterno na prática do aleitamento materno
}

\author{
Paternal incentive in the practice of breastfeeding \\ Incentivo paterno en la práctica de lactancia materna
}

Suzana Lins da Silva ${ }^{1 *}$, Maria de Fátima Costa Caminha ${ }^{1}$, Camila Carvalho dos Santos ${ }^{1}$, Matheus Lauriano da Silva Cruz ${ }^{2}$, Thais de Albuquerque Corrêa ${ }^{3}$, Déborah Lemos Freitas ${ }^{1}$, Rayanne Victória Araújo Lins Rocha ${ }^{3}$, Elisama da Paz Oliveira Lima ${ }^{3}$, Luciana da Silva Barreto ${ }^{3}$, Malaquias Batista Filho ${ }^{1}$.

\section{RESUMO}

Objetivo: Identificar a frequência do incentivo paterno para a prática do Aleitamento Materno (AM). Métodos: Estudo transversal, recorte do inquérito "Saúde, nutrição e serviços assistenciais numa população carente do Recife", com coleta de dados no período de julho a outubro/2015. A população do estudo atual foi representada pelas mães das 310 crianças de zero a três anos usuárias das duas Unidades Básicas de Saúde existentes na comunidade estudada. Selecionadas as variáveis de interesse e elaborado banco "ad hoc". Os dados foram analisados no programa Stata 12.1. O inquérito foi aprovado pelo Comitê de Ética em Pesquisa Abordando Seres Humanos (CEP). Resultados: Pouco mais da metade das mães $(57,7 \%)$ recebeu incentivo do pai da criança ao AM. A maioria (92,9\%) havia realizado pré-natal, porém 93,9\% não receberam orientação sobre AM. Metade $(55,1 \%)$ recebeu visita domiciliar na primeira semana de vida. Prevaleceu a faixa etária entre 20 a 35 anos $(72,4 \%)$. Pouco mais da metade $(64,0 \%)$ possuía abaixo de 12 anos de estudo e a maioria (72,6\%) pertencia a classe social C. Conclusão: Os dados deste estudo apontam para o reconhecimento do pai como importante influenciador no processo de AM, pois pode ser um aliado e estimulador dessa prática.

Palavras-chave: Incentivo, Aleitamento materno, Período pós-parto, Paternidade, Cuidado pré-natal.

\begin{abstract}
Objective: To identify the frequency of paternal incentive to practice breastfeeding. Methods: Cross-sectional study, part of the research "Health, nutrition and assistance services in the favela population of Recife", with data collection from July to October / 2015. The population was represented by mothers of 310 children from zero to three years old, users of the two Basic Health Units of the studied favela. An "ad hoc" bank was created. The data were analyzed using the Stata 12.1 program. The research was approved by the IMIP Research Ethics Committee for Human Beings (CEP / IMIP). Results: $57.7 \%$ of mothers received encouragement from the child's father to breastfeed justified by: paternal absence, attitudes of indifference towards the mother and distrust towards paternity. The majority $(92.9 \%)$ received prenatal care, however $93.9 \%$ did not receive guidance on breastfeeding. $55.1 \%$ received home visits in the first week of life. The 20 to 35 age group prevailed. $64.0 \%$ had less than 12 years of study and $72.6 \%$ belonged to social class $\mathrm{C}$. Conclusion: The data in this study point to the recognition of the father as an important influencer in the breastfeeding process, as he can be a ally and stimulator of this practice.
\end{abstract}

Keywords: Motivation, Breast feeding, Postpartum period, Paternity, Prenatal care.

\section{RESUMEN}

Objetivo: Identificar la frecuencia de incentivo paterno para practicar la lactancia materna (LM). Métodos: Estudio transversal, parte de la encuesta "Servicios de salud, nutrición y asistencia en una población de tugurios en Recife", con recolección de datos de julio a octubre / 2015. La población del presente estudio estuvo representada por las madres de 310 niños de cero a tres años, usuarias de las dos Unidades Básicas de Salud de la barriada estudiada. Se creó un banco "ad hoc". Los datos se analizaron utilizando el programa Stata 12.1. La encuesta fue aprobada por el Comité de Ética en Investigación sobre Seres Humanos del IMIP

1 Instituto de Medicina Integral Prof. Fernando Figueira (IMIP), Recife - PE. *E-mail: suzana.lins@imip.org.br

2 Hospital da Restauração Governador Paulo Guerra, Recife - PE.

${ }^{3}$ Faculdade Pernambucana de Saúde (FPS), Recife - PE. 
(CEP / IMIP). Resultados: 57,7\% recibieron estímulo del padre del niño para amamantar. 92,9\% había recibido atención prenatal, sin embargo, el 93,9\% no recibió orientación sobre LM. 55,1\% recibió visitas domiciliarias en la primera semana de vida. Prevaleció el grupo de edad de 20 a 35 años (72,4\%). 64,0\% tenía menos de 12 años de estudio y 72,6\% pertenecía a la clase social C. Conclusión: Los datos de este estudio apuntan al reconocimiento del padre como un influyente importante en el proceso de MA ya que puede ser un aliado y estimulador de esta práctica.

Palabras clave: Incentivo, Lactancia materna, Período posparto, Paternidad, Atención prenatal.

\section{INTRODUÇÃO}

O aleitamento materno é um processo que possibilita o melhor desenvolvimento da criança, promovendo a prevenção de possíveis problemas de saúde, assim como o desenvolvimento de uma imunidade mais resistente para o bebê. Seu estímulo diminui os riscos de várias doenças e aumenta a qualidade de vida da criança como também proporciona vínculos entre a genitora e sua família. É uma experiêcia individual de cada mulher, e, em algumas mães, acarreta alguns momentos desafiadores, tais como: dor e desconforto, ferimento dos seios e também questões psicológicas em todo o processo, porém, é fato que os pontos positivos superam todas as possíveis adversidades. Tendo em vista tais pontos, é essencial uma rede de apoio concreta e sólida em todo o processo de amamentação (MINISTÉRIO DA SAÚDE, 2015a; MINISTÉRIO DA SAÚDE, 2015b).

Segundo o Ministério da Saúde (MS), o aleitamento deve ser iniciado após o nascimento, assim como ser exclusivo durante os primeiros seis meses de vida e, posteriormente, combinado a outros alimentos durante dois anos ou mais. Todavia, é importante ressaltar que o Ministério da Saúde frisa que a introdução de outros nutrientes antes dos seis meses pode ocasionar prejuízo à saúde das crianças (MINISTÉRIO DA SAÚDE, 2015b).

Nesta perspectiva, é importante lembrar como o profissional enfermeiro vai mediar o incentivo ao aleitamento materno durante o pré-natal, esclarecendo a futura puérpera os benefícios da amamentação adequada, como também os riscos que o recém-nascido poderá desenvolver caso esta "ferramenta natural" não seja utilizada de forma correta, evitando assim o risco de desistência por parte da mesma. Este acolhimento profissional é essencial para instruir, acalmar e assegurar o aleitamento de forma correta por parte da genitora, assim como, fundamental para incluir o pai durante o desenvolvimento de todo o processo (COSTA FS, et al., 2019)

Por meio do Sistema Único de Saúde (SUS), o governo tem instituído e apoiado programas que possibilitam e empoderam a atuação da mulher na amamentação. Eles são essenciais para o incentivo e realização do aleitamento materno, além de fortalecer esta prática. São elas estratégias que promovem, protegem e apoiam o aleitamento materno: Método Canguru, a Rede Brasileira de Banco de Leite Humano, a Estratégia Amamentar e Alimentar o Brasil, a Rede Cegonha e a inclusão de ações voltadas para as mulheres trabalhadoras que amamentam (ASSOCIAÇÃO BRASILEIRA DE EMPRESAS DE PESQUISA (ABEP), 2014; MINISTÉRIO DA SAÚDE, 2015b; GONTIJO TL, et al., 2012; PASSANHA A, et al., 2013; GIUGLIANI ERJ, 2002).

Os resultados dessas estratégias no Brasil, podem ser demonstrados através de estudos científicos (SILVA VAAL, et al., 2018; OLIVEIRA DS, et al., 2017; BOCCOLINI CS, et al., 2017). A pesquisa de Boccolini CS, et al. (2017) analisou a tendência dos indicadores de aleitamento materno no Brasil nas últimas três décadas, proveniente de informações de 1986, 1996, 2006 e 2013 da Pesquisa Nacional de Saúde, apresentando como resultado tendência ascendente até 2006, entretanto, com estabilização entre 2006 e 2013 (BOCCOLINI CS, et al., 2017). Os resultados de Boccolini CS, et al. (2017), surgem como sinal de alerta, segundo considerações dos próprios autores, para favorecimento da avaliação e revisão das políticas e programas de promoção, proteção e apoio ao aleitamento (BOCCOLINI CS, et al., 2017).

Além do âmbito hospitalar e da assistência básica, é conhecida a importância do apoio familiar. Dentro desse cenário, é reconhecido que as atitudes e ações paternas podem afetar positiva ou negativamente na 
intenção das mães de amamentar, duração da amamentação e amamentação exclusiva (ROLL CL, CHEATER F, 2016; SILVEIRA FJFD, et al., 2018). Além do apoio paterno, é necessária uma forte rede de apoio para proporcionar confiança, segurança e otimismo. Essa assistência favorece um suporte psicoafetivo à cada mulher, contribuindo, desta forma, para o seu crescimento pessoal e construção de um afeto mais consolidado ao seu bebê. O apoio físico e psicológico é extremamente importante neste momento singular, pois as emoções pertencentes a elas costumam oscilar durante todo o processo, visto que as alterações de humor são decorrentes tanto de hormônios como de experiências negativas ao decorrer da amamentação (SILVA BT, et al., 2012).

Por outro lado, o que se observa é a sua invisibilidade por parte dos profissionais de saúde, como é demonstrado em estudo qualitativo que objetivou entender o conhecimento paterno voltado às tribulações durante o aleitamento materno, e que após sua observação surgiram algumas ideias centrais, tais como: a compreensão contra a incompreensão sobre o aleitamento; a equipe que não inclui o pai durante o processo e sentimentos novos e aflorados frente às dificuldades na amamentação. Em contrapartida, foram perceptíveis a disposição e a vontade dos pais em participar e apoiar suas companheiras, porém não são inseridos e encorajados pela equipe profissional de saúde (PINTO KRTF, et al., 2018).

É comum encontrar pais que valorizam e consideram a amamentação saudável e natural para os bebês. Mas, por outro lado, pelas dificuldades das suas parceiras, percebem que pode ser uma prática potencialmente prejudicial para as mães, uma vez que os problemas da amamentação podem afetar também o ambiente familiar, assim, desestabilizando toda a rede de apoio (HANSEN E, et al., 2018). Nos espaços urbanos e rurais mais pobres, o segmento materno-infantil caracteriza-se por sua vulnerabilidade, representando o efeito interativo de condições biológicas peculiares deste grupo com a superposição de fatores sociais e ambientais desfavoráveis (SANTOS FS, et al., 2016). A maioria das doenças que resultam em mortes infantis poderiam ser evitadas através de medidas de promoção, proteção e apoio ao aleitamento materno (VICTORA CG, et al., 2016).

Tomando como princípio que um pai instruído e interativo durante a amamentação poderá ser um integrante dinâmico e participativo em todo o processo, assim, reduzindo o desmame precoce, o estudo atual objetivou identificar a frequência do incentivo paterno para a prática do aleitamento materno em um ambiente de vulnerabilidade como um aglomerado urbano subnormal do nordeste brasileiro.

\section{MÉTODOS}

Estudo transversal, recorte do inquérito "Saúde, nutrição e serviços assistenciais numa população carente do Recife", com coleta de dados entre julho e outubro de 2015, realizado em um aglomerado urbano subnormal da cidade de Recife. Adotando cobertura censitária, o inquérito estudou todas as crianças de 0 a 36 meses que eram registradas pelos Agentes Comunitários de Saúde das duas Unidades Básicas de Saúde da localidade.

A população do estudo atual é representada por todas as mães das 310 crianças de zero a três anos residentes na comunidade, usuárias das duas Unidades Básicas de Saúde existentes na localidade. A amostra possui caráter censitário, com o tamanho equivalente, por definição ao tamanho de toda a população de crianças até três anos.

Foram incluídas todas as mães de crianças de zero a três anos de idade disponíveis no banco de dados e excluídas as mães de crianças do banco de dados com falta de informações sobre o incentivo ao aleitamento materno.

As crianças foram identificadas através de registros e prontuários com os Agentes Comunitários de Saúde (ACS) nas unidades de Saúde da comunidade. Foram realizadas visitas em suas residências, visando à aceitação das mães e/ou responsáveis legais das crianças na participação na pesquisa. As enfermeiras das duas Unidades de Saúde forneciam antecipadamente a lista de crianças quando iam cumprir atividades de puericultura, sendo então também aproveitada esta oportunidade para captação dos participantes. As famílias que aceitaram participar da pesquisa assinaram o Termo de Consentimento Livre e Esclarecido (TCLE). 
As variáveis maternas estudadas foram idade, estado civil, instrução, classe social, realização de prénatal, número de consultas no pré-natal, orientação durante o pré-natal sobre aleitamento materno, estímulo a presença do companheiro nas consultas no pré-natal, visita domiciliar na primeira semana após a alta da maternidade, incentivo ao aleitamento materno pelo pai da criança, e se o pai não incentivou, o que ele referia a mãe da criança sobre a prática do aleitamento materno.

Os dados do estudo original foram coletados através de entrevistas com as mães das crianças ou cuidadores em seus domicílios ou nas unidades básicas de saúde e posteriormente digitados no Programa Excel com dupla entrada e validados no Epi-Info 3.5.3.

Para os objetivos de interesse do estudo atual, foi formado um arquivo ad hoc seletivamente construído a partir das informações digitadas e validadas na pesquisa primária. As variáveis foram recodificadas quando necessário à análise estatística, de acordo com os objetivos propostos. Os dados do estudo atual foram analisados utilizando o programa Stata 12.1. Os dados categóricos foram descritos através de tabelas de distribuição de frequências.

O inquérito "Saúde, nutrição e serviços assistenciais numa comunidade do Recife", de onde foi realizado recorte para o estudo atual foi aprovado pelo Comitê de Ética em Pesquisa envolvendo Seres Humanos, CAAE número 07246912.6.0000.5201.

\section{RESULTADOS}

A frequência do incentivo paterno para a prática do aleitamento materno foi de 57,7\%. A Tabela 1 mostra os valores absolutos e percentuais referente as características sociodemográficas e obstétricas maternas. Do total das 310 mães das crianças que participaram do estudo prevaleceu a idade entre 20 a 35 anos $(72,4 \%)$. Pouco mais da metade $(64,0 \%)$ possuía abaixo de 12 anos de estudo e a maioria $(72,6 \%)$ pertencia a classe social C. Com relação às informações obstétricas, a maioria das mães $(92,9 \%)$ haviam realizado o pré-natal e $80,9 \%$ tiveram 6 ou mais consultas durante toda a gestação.

Tabela 1 - Variáveis sociodemográficas e obstétricas das mães de crianças menores de três anos em unidades básicas de saúde em uma comunidade no nordeste do Brasil, 2015.

\begin{tabular}{lc}
\hline Variáveis & $\mathbf{N}(\%)$ \\
\hline ldade materna $(\mathbf{N}=\mathbf{3 1 0})$ & \\
\hline$<20$ anos & $51(16,5)$ \\
20 a 35 anos & $230(74,2)$ \\
$>=36$ anos & $29(9,4)$ \\
\hline Anos de estudo materno $\left(\mathbf{N}=\mathbf{3 0 8}^{\star}\right)$ & $111(36,0)$ \\
\hline$>12$ & $197(64,0)$ \\
\hline 12 & \\
\hline Classe Social da família** $(\mathbf{N}=\mathbf{3 1 0})$ & $15(4,8)$ \\
\hline B & $225(72,6)$ \\
D e E & $70(22,6)$ \\
\hline Realização de pré-natal $\left(\mathbf{N}=\mathbf{3 0 8}^{\star}\right)$ & \\
\hline Sim & $286(92,9)$ \\
$\quad$ Não & $22(7,1)$ \\
\hline Número de consultas no pré-natal $\left(\mathbf{N}=\mathbf{2 7 7}^{\star}\right)$ & \\
\hline$<6$ consultas & $53(19,1)$ \\
$\geq 6$ consultas & $224(80,9)$ \\
\hline
\end{tabular}

Legenda: *A amostra variou devido a ausência de respostas. Fonte: Silva SLD, et al., 2021.

A Tabela 2 apresenta as frequências de orientação e incentivo ao aleitamento materno nas crianças. Destaca-se que $93,9 \%$ das mães não receberam orientação durante o pré-natal sobre aleitamento materno e cerca de metade $(55,1 \%)$ recebeu visita domiciliar na primeira semana de vida. Como se observa, $57,7 \%$ das mães recebeu incentivo do pai da criança ao aleitamento materno. A falta de incentivo dos pais sobre a prática da amamentação justificava-se por ausência paterna, atitudes de indiferença na relação com a mãe e desconfiança em relação a paternidade. 
Tabela 2 - Variáveis relacionadas a orientação e incentivo ao aleitamento materno de crianças menores de três anos em unidades básicas de saúde em uma comunidade no nordeste do Brasil, 2015.

\begin{tabular}{lc}
\hline Variáveis & $\mathbf{N}(\%)$ \\
\hline Orientação durante o pré-natal sobre aleitamento materno (N=277*) & 17 \\
\hline Sim & $(6,1)$ \\
Não & $260(93,9)$ \\
\hline Recebeu visita domiciliar na primeira semana de vida da criança (N=303*) & $167(55,1)$ \\
\hline Sim & $136(44,9)$ \\
Não & $167(57,7)$ \\
\hline Recebeu incentivo do pai da criança ao aleitamento materno (N=289*) & $122(42,3)$ \\
\hline Nim & $15(11,7)$ \\
\hline Motivo paterno sobre não incentivar a prática do aleitamento materno (N= 128*) & $107(83,6)$ \\
\hline Ausente & 6 \\
Atitudes de indiferença na relação com a mãe & $(4,7)$ \\
Desconfiança em relação a paternidade &
\end{tabular}

Legenda: *A amostra variou devido à ausência de respostas. Fonte: SILVA SLD, et al., 2021.

\section{DISCUSSÃO}

A prática do aleitamento materno é de grande relevância para a saúde da criança, repercutindo em toda sua vida adulta, onde o incentivo paterno, através de suas atitudes e ações, assume um papel de fundamental importância no apoio materno para amamentar, tendo em vista que a presença do parceiro estimula as mães a prolongar a lactação (SILVEIRA FJFD, et al., 2018). Entretanto, no estudo atual, um pouco mais da metade das mães recebeu incentivo do pai da criança para o aleitamento materno. A falta de incentivo dos pais sobre a prática da amamentação justificou-se por ausência paterna, atitudes de indiferença na relação com a mãe e desconfiança em relação a paternidade. A pouca participação paterna no processo de amamentação gera sentimentos de frustração na mãe, além de insatisfação com o processo, sensação de abandono, experiência negativa e, também, exaustão, podendo até influenciar a uma depressão pós-parto.

O acompanhamento do pai na consulta pré-natal possibilita um maior conhecimento sobre as mudanças durante toda a gravidez, reduzindo, assim, medos e angústias provocadas pelas dúvidas sobre como proceder ao nascimento do bebê. Além disso, cuidar do bebê é uma responsabilidade do casal, e, quando os pais são devidamente orientados e encorajados, eles irão amparar suas companheiras a dominar e vencer qualquer empecilho que possa manifestar-se durante o processo de amamentação. Para isso, a atuação dos profissionais de saúde no desenvolvimento desta prática é de fundamental importância (LIMA JP, et al., 2017).

Um estudo observacional e transversal realizado com 351 casais constatou que $92,02 \%$ dos pais expôs não ter recebido dos profissionais de saúde esclarecimentos ou explicações sobre a amamentação. Mesmo que esta prática seja um ato extremamente íntimo entre mãe e filho, é essencial o encorajamento e estímulo por parte do profissional a fim de ajudar todo o curso desta experiência singular (SILVEIRA FJFD, et al., 2018).

Artigo que teve como objetivo analisar o conhecimento e as atitudes paternas acerca do aleitamento materno evidenciou que $51,4 \%$ dos homens-pais entrevistados acompanharam suas companheiras durante as consultas de pré-natal, enfatizando a importância do aleitamento materno. Porém, a falta de orientações durante as consultas ainda foi escassa, sendo uma situação preocupante visto que o estudo é bastante recente, além do mais, a atuação dos profissionais no aleitamento é essencial para uma promoção de qualidade (BRÁULIO TIC, et al., 2021).

Apenas $4,27 \%$ dos pais tinham o conhecimento da duração do aleitamento materno aconselhado pela Organização Mundial de Saúde (OMS) e o MS. Além disso, 56,98\% dos pais explanaram que não dialogavam sobre alimentação infantil com a mãe da criança (SILVEIRA FJFD, et al., 2018). Tal achado evidencia que não há muito incentivo e ensinamento sobre os benefícios da amamentação, o que se torna um grande problema entre os casais, pois, por não terem o conhecimento sobre tal temática, não dialogam sobre o referido assunto entre si, prejudicando assim a duração e prática efetiva do aleitamento. 
Embora outra pesquisa revelasse que os pais estão interessados em aleitamento materno e querem ser envolvidos na preparação e apoio da amamentação, é notório o interesse pelas questões práticas, devendo o profissional de saúde demonstrar como participar os cuidados, prestando apoio a suas parceiras (RÊGO RM, et al., 2016).

É importante salientar que esta pesquisa demonstrou que apenas $6,1 \%$ das genitoras tiveram instruções no decurso de todo o pré-natal. Esta porcentagem também está exposta de forma semelhante em outros trabalhos nacionais, como um estudo do Rio de Janeiro no qual somente $28,7 \%$ tiveram elucidações efetivas sobre o aleitamento materno e também sobre o parto (DOMINGUES RMSM, et al., 2012). Cabe ainda apresentar aqui um estudo desempenhado na região nordeste brasileira onde $89,8 \%$ das gestantes foram aconselhadas e atualizadas sobre o AM durante o pré-natal, onde, o recebimento desses esclarecimentos associou-se a uma prevalência maior do aleitamento (TENÓRIO MCDS, et al., 2018).

A falta de orientação e incentivo pode ser fator de risco para o desmame precoce, e, com isso, predispõe o bebê ao surgimento de possíveis doenças advindas de fórmulas nutricionais, como diarreia (podem ser decorrentes de intolerâncias alimentares), infecções respiratórias e otite média (as fórmulas não oferecem anticorpos para o bebê, deixando-o vulnerável à infecções), obesidade (a maioria das fórmulas possuem uma grande quantidade de proteínas, favorecendo a obesidade precoce), problemas no desenvolvimento cognitivo e também defeitos ortodônticos (decorrentes do uso de mamadeiras) (STUEBE A, 2009; TESTON EF, et al., 2018).

Ademais, as mães também podem sofrer consequências do desmame precoce, o que poderá aumentar 0 risco de câncer de mama e de ovário, bem como obesidade, diabetes tipo 2, síndromes metabólicas (o leite humano previne síndromes metabólicas pois demanda de cargas metabólicas altas para amamentar) e doenças cardiovasculares (LIMA APC, et al., 2018; SILVA D, et al., 2017; STUEBE A, 2009).

Entender o conhecimento prévio dos pais acerca da amamentação é crucial para reconhecer todos os anseios e preocupações da mãe e também do pai, isso irá direcionar a educação e o ensinamento do profissional para com o casal voltado para as necessidades específicas de cada um. Para isso, algumas perguntas mais diretas podem ajudar à nortear a assistência, como: "o que você sabe sobre amamentação?" e "quais seus medos e angústia acerca desta prática?". De acordo com as respostas de cada casal, o profissional irá averiguar qual o nível de entendimento tanto da mãe, do pai como também de toda a família (se possível) acerca do aleitamento materno (STUEBE A, 2009; TESTON EF, et al., 2018).

Neste sentido, destaca-se que o profissional de enfermagem deve ser um incentivador e um facilitador da prática da amamentação, durante o pré-natal e na visita domiciliar, valendo-se de um conjunto de medidas com informações adequadas e acessíveis ao entendimento e ao conhecimento da mulher nutriz, pois, é de responsabilidade dos profissionais de saúde reconhecer e conduzir adequadamente problemas relacionados à amamentação. Ainda, mães em risco de não amamentar e seus parceiros devem ser alvos de ações de promoção do aleitamento materno, tendo em vista que essas atividades irão levar mais conhecimento à todos os pais que sentem dificuldade durante o curso dessa prática (COCA KP, et al., 2018; LUSTOSA E e LIMA $\mathrm{RN}, 2020)$.

No presente estudo, cerca de metade recebeu visita domiciliar na primeira semana de vida. A visita domiciliar é um momento oprtuno para o enfermeiro demonstrar a nutriz como se dá o manejo clínico da amamentação, orientar quanto as dúvidas e estimular o apoio familiar, principalmente o envolvimento do pai, favorecendo assim o aleitamento materno exclusivo até os 6 meses de idade. Portanto, cabe ao profissional averiguar como se encontra aquela puérpera e seu parceiro em relação à amamentação naquele momento, visto que, isso irá prevenir problemas e direcionar melhor a prática correta. Ademais, é importante ser direto no momento da visita domiciliar e perguntar ao casal como está indo o processo de amamentação, visto que é um momento propício para enfatizar as recomendações da amamentação exclusiva durante os primeiros 6 meses e oferecer dicas e instruções sobre a técnica correta (LUSTOSA E e LIMA RN, 2020).

No que se refere à condição socioeconômica, as evidências científicas mostram que mulheres com mais de oito anos de estudo, idade acima de 30 anos e com renda melhor são mais propensas para o sucesso da prática do aleitamento materno, assim como uma duração mais efetiva (MARGOTTI E e MARGOTTI W, 2017; 
TEWABW T, et al., 2016). No grupo estudado, por tratar-se de estudo realizado em aglomerado suburbano, a maioria pertencia a classe $\mathrm{C}$ e mais da metade possuía menos de 12 anos de estudo. Esses dados apontam para uma relação de menor acesso a informações e baixo reconhecimento sobre os benéficos do aleitamento materno. Visto que a educação está diretamente ligada ao aprendizado e conhecimento, muitos casais que não tiveram oportunidade de estudos podem ser prejudicados no entendimento da prática, seus benefícios e particularidades.

\section{CONCLUSÃO}

Os dados deste estudo apontam para o reconhecimento do pai como um importante influenciador no processo de aleitamento materno por ser um aliado e estimulador dessa prática e que deve caminhar lado a lado de sua companheira e do seu bebê. Cabe aos enfermeiros incluir a presença paterna em todo o processo de amamentação e incentivá-lo a participar desse momento vital para a família. Além disso, é extremamente significativo capacitar os futuros profissionais para que os mesmos estejam aptos à ensinar, demonstrar e ajudar o casal com essa prática que envolve mais aspectos positivos do que possíveis adversidades, além do mais, com profissionais qualificados e mais humanizados, a prática do processo de amamentação poderá abarcar um número maior de mães e pais que estão iniciando esta jornada.

\section{REFERÊNCIAS}

1. ASSOCIAÇÃO BRASILEIRA DE EMPRESAS DE PESQUISA (ABEP). Critério de classificação econômica. Brasil, 2014. Disponível em: https://www.abep.org/criterio-brasil. Acessado em: 15 de novembro de 2021.

2. BRÁULIO TIC, et al. Conhecimento e atitudes paternas acerca da importância do aleitamento materno. Escola Anna Nery, 2021; 25.

3. BOCCOLINI CS, et al. Tendência de indicadores do aleitamento materno no Brasil em três décadas. Rev. Saúde Pública, 2017; 51: 108.

4. COCA KP, et al. de medidas para o incentivo do aleitamento materno exclusivo intra-hospitalar: evidências de revisões sistemáticas. Rev. Paul. Pediatr. (Ed. Port., Online), 2018; 36(2): 214-220.

5. COSTA FS, et al. Promoção do aleitamento Materno no Contexto da Estratégia de Saúde da Família. Rev Rede de Cuidados em Saúde, 2019; 13(1): 1-15.

6. DOMINGUES RMSM, et al. Avaliação da adequação da assistência pré-natal na rede SUS do Município do Rio de Janeiro, Brasil. Cad Saúde Pública, 2012; 28(3): 425-37.

7. GIUGLIANI ERJ. Rede Nacional de Bancos de Leite Humano do Brasil: tecnologia para exportar. J. Pediatr (Rio J), 2002; 78(3): 183-4.

8. GONTIJO TL, et al. Avaliação da implantação do Método canguru por gestores, profissionais e mães de recémnascidos. Cad. Saúde Pública, 2012; 28(5): 935-44.

9. HANSEN E, et al. 'They're born to get breastfed'- how fathers view breastfeeding: a mixed method study. BMC Pregnancy Childbirth, 2018; 18(1): 238.

10. LIMA APC, et al. A prática do aleitamento materno e os fatores que levam ao desmame precoce: uma revisão integrativa. J. of Health \& Biological Sciences, 2018; 6(2): 189-196.

11. LIMA JP, et al. Involvement of fathers in the breastfeeding process. Cogitare Enferm, 2017; 22(1): 01-07.

12. LUSTOSA E, LIMA RN. Importância da enfermagem frente à assistência primária ao aleitamento materno exclusivo na atenção básica. Rev Brasileira Interdisciplinar de Saúde, 2020; 3(1): 1-5.

13. MARGOTTI E, MARGOTTI W. Fatores relacionados ao Aleitamento Materno Exclusivo em bebês nascidos em hospital amigo da criança em uma capital do Norte brasileiro. Saúde em Debate, 2017; 41: 860-871.

14. MINISTÉRIO DA SAÚDE. Mulher trabalhadora que amamenta, 2015a. Disponível em: https://bvsms.saude.gov.br/bvs/publicacoes/cartilha_mulher_trabalhadora_amamenta.pdf. Acessado em: 15 de novembro de 2021.

15. MINISTÉRIO DA SAÚDE. Saúde da criança: aleitamento materno e alimentação complementar, 2015b. Disponível em: http://189.28.128.100/dab/docs/portaldab/publicacoes/saude_crianca_aleitamento_materno_cab23.pdf. Acessado em 15 de novembro de 2021.

16. OLIVEIRA DS, et al. Duração do aleitamento materno e fatores associados entre 1960 e 2000. J. Pediatr, 2017; 93(2): 130-135.

17. PASSANHA A, et al. Implantação da Rede Amamenta Brasil e prevalência de aleitamento materno exclusivo. Rev. Saúde Publica, 2013; 47(6): 1141-1148. 
18. PINTO KRTF, et al. Dificuldades na amamentação: sentimentos e percepções paternas. J. of Nursing and Health, 2018; (8)1:1-12.

19. RÊGO RM, et al. Paternidade e amamentação: mediação da enfermeira. Acta Paulista de Enfermagem, 2016; 29(4): 374-80.

20. ROLL CL, CHEATER F. Expectant parents' views of factors influencing infant feeding decisions in the antenatal period: a systematic review. Int J Nurs Stud, 2016; 60: 145-55.

21. SANTOS FS, et al. Aleitamento Materno e Diarreia Aguda entre Crianças Cadastradas na Estratégia Saúde da Família. Texto Contexto Enferm, 2016; 25(1): 0220015.

22. SILVA BT, et al. Apoio paterno ao aleitamento materno: uma revisão integrativa. Rev Paulista Pediatr, 2012; 30(1): $122-130$.

23. SILVA D, et al. Aleitamento materno: causas e consequências do desmame precoce. Unimontes Científica, 2017; 19(2): 146-157.

24. SILVA VAAL, et al. Maternal breastfeeding: indicators and factors associated with exclusive breastfeeding in a subnormal urban cluster assisted by the Family Health Strategy. J. Pediat, 2018; 95(3): 298-305.

25. SILVEIRA FJFD, et al. Conhecimento dos pais sobre o processo de aleitamento materno em mães de uma maternidade pública em Belo Horizonte, MG. Rev. Médica de Minas Gerais, 2018; 28: 1969.

26. STUEBE A. The risks of not breastfeeding for mothers and infants. Reviews in obstetrics and gynecology, 2009; 2 (4): 222.

27. TENÓRIO MCDS, et al. Factors associated with the lack of breastfeeding upon discharge from hospital in a public maternity facility in maceió, alagoas, Brazil. Cienc. e Saúde Coletiva, 2018; 23(11): 3547-56.

28. TESTON EF, et al. Aleitamento materno: percepção do pai sobre seu papel. Revista de Enfermagem do Centro-Oeste Mineiro, 2018; 8.

29. TEWABE T, et al. Exclusive breastfeeding practice and associated factors among mothers in Motta town, East Gojjam zone, Amhara Regional State, Ethiopia, 2015: a cross-sectional study. International breastfeeding jornal, 2016; 12(1): 12.

30. VICTORA CG, et al. Amamentação no século 21: epidemiologia, mecanismos, e efeitos ao longo da vida. Epidemiologia em Serviços de Saúde, 2016; 25(1): 1-24. 